\title{
Editorial
}

\section{Neurological Intensive Care}

Critical care medicine has seen tremendous advances over recent years. The specialty now accounts for improved patient survival in greater numbers and with ever increasing complexities of care requirements. These are patients commonly present with associated neurological problems [1].

The multicenter pan-European SOAP study [2] prospectively collated demographic, co-morbidity, clinical and laboratory data on new ICU admissions over a two-week period. In 16\% of admissions, neurological dysfunction represented the third most common indication for admission after cardiovascular and respiratory aetiologies (32\% and 19\% respectively).

Another observational study of 1850 critically ill patients admitted to a medical intensive care unit revealed 92 patients to be primarily admitted with neurological compromise (5\%), and a further 216 went on to develop neurological complications (12.3\%) [3]. The most common of these included seizures, metabolic or hypoxic-ischemic encephalopathy and cerebral vascular events (CVE). Evidence suggests that these patients have 2.5 fold increased ICU admission and a two-fold longer overall hospital stay [3].

Needless to say the ICU physician requires a high degree of expertise in neurology. Routine ICU consultations include assessments of failure to regain or impaired consciousness, seizures, confusion, involuntary or weak muscle function, post CVE deficits, inability to ventilator wean, prognosis and declaration of brain death.

Specialized neurological intensive care units (neuro-ICU's) originally evolved from the respiratory support facilities in the epidemic era of polio myelitis. Today, they are able to offer focused, appropriate and rapid treatment modalities to their patients. Their spectrum comprises neurological, respiratory, cardiovascular, and disease-specific therapies such as plasma exchange, immunoabsorption, intracranial pressure monitoring and treatment, hypothermia and many more [5]. Development continues with interventional neuroradiology as a contemporaneous new frontier in the treatment of patients with intracranial stenosis, vessel occlusion or vascular malformation [7].

The care of the critically ill neurological patient is not simply a combination of critical care and neurological assessment [8], but an integrated treatment plan specific for neurological disease. Neuro-ICU remains a domain of neurology whereby the challenge is the application of neurological pathophysiology theory in the modern critical care setting.

This special issue of 'Neurological Intensive Care' is a comprehensive scientific and evidence based overview of the relevant topics. It presents a selection of articles dealing with a variety of neurological complications encountered within the general ICU environment. It also highlights specific neurological diseases and their management strategies relevant to neuro-ICU. It should prove of interest to intensivists of all specialties.

\section{REFERENCES}

[1] Bleck TP. Neurological disorders in the intensive care unit. Semin Respir Crit Care Med 2006; 27: 201-9.

[2] Vincent J-L, Sakr Y, Sprung CL, et al. Sepsis in European intensive care units: Results of the SOAP study. Crit Care Med 2006; $34: 34453$.

[3] Bleck TP, Smith MC, Pierre-Louis SJ, Jares JJ, Murray J, Hansen CA. Neurologic complications of critical medical illnesses. Crit Care Med 1993; 21 : $98-103$.

[4] Razvi SSM, Bone I. Neurological consultations in the medical intensive care unit. J Neurol Neurosurg Psychiatr 2003; 74 Suppl 3: iiil6-23.

[5] Howard RS. Neurological problems on the ICU. Clin Med 2007; 7: 148-53.

[6] Ropper AH. Neurological intensive care. Ann Neurol 1992; 32: 564-9.

[7] Connolly ES, Lavine SD, Meyers PM, Palistrandt D, Parra A, Mayer SA. Intensive care unit management of interventional neuroradiology patients. Neurosurg Clin N Am 2005; 16: 541-5.

[8] Wijdicks EFM. Changing aspects of care in critically ill neurologic patients. Rev Neurol Dis 2008; 5: 1349.

\author{
Albrecht Günther \\ (Co-Guest Editor) \\ Hans Berger Clinic for Neurology \\ Jena University Hospital \\ Friedrich-Schiller-University Jena \\ Erlanger Allee 101 \\ D-07747 Jena \\ Germany
}

Hubertus Axer

(Guest Editor)

Hans Berger Clinic for Neurology Jena University Hospital Friedrich-Schiller-University Jena Erlanger Allee 101 D-07747 Jena Germany

E-mail: Hubertus.Axer@med.uni-jena.de

(C) Günther and Axer.; Licensee Bentham Open.

This is an open access article licensed under the terms of the Creative Commons Attribution Non-Commercial License (http://creativecommons.org/licenses/by-nc/ 3.0/) which permits unrestricted, non-commercial use, distribution and reproduction in any medium, provided the work is properly cited. 